\title{
ON A SET OF POSTULATES WHICH SUFFICE TO DEFINE A
}

\author{
NUMBER-PLANE*
}

BY

ROBERT L. MOORE

In this paper I propose to show that any plane satisfying Veblen's Axioms I-VIII, XI of his System of axioms for geometry $\dagger$ is a number-plane, or in other words that any plane $V$ satisfying these axioms contains a system of continuous curves such that, with reference to these curves regarded as straight lines, the plane $V$ is an ordinary euclidean plane.

In consequence, any discussion of analysis situs based on these axioms (as, for example, Veblen's proof $\ddagger$ of the theorem that a jordan curve divides its plane into just two parts) is no more general than one based on analytic hypotheses. This does not contradict the fact that the geometry based on axioms I-VIII, XI is much more general than euclidean geometry in the sense that the curves with respect to which the plane $V$ is euclidean are not necessarily the straight lines referred to in these axioms.

My argument also furnishes the answer to a problem proposed by Veblen in another paper. He states this problem as follows.§

"An interesting situation is obtained by introducing a postulate of uniformity among the hypotheses of plane analysis situs (cf. p. 84 of this volume). If the postulate is applied to the straight line, the line is necessarily a continuum but it is not obvious that other curves are. If it is applied to the plane, the segments $\sigma_{\nu P}$ in this case being triangular regions, all continuous curves are continua, but it is not obvious that there is a one-to-one reciprocal correspondence between the plane and a set of number-pairs."

Without adding any postulate of uniformity to Veblen's Axioms I-VIII,

* A part of this paper, under the title Concerning so-called non-metrical analysis situs, was presented to the Society, April 27, 1912.

†O. Veblen, $A$ system of axioms for geometry, these Transactions, vol. 5 (1904), pp. 343-384.

$\ddagger \mathrm{O}$. Veblen, Theory of plane curves in non-metrical analysis-situs, these $\mathrm{Tr}$ a $\mathrm{n}$ s a cti o $\mathrm{s}$, vol. 6 (1905), pp. 83-98. See also vol. 14, p. 65.

$\$ 0$. Veblen, Definition in terms of order alone in the linear continuum and in well-ordered sets, these Transactions, vol. 6 (1905), pp. 165-171. 
XI, Lennes* has shown that all continuous curves in $V$ are continua. He does not however show that there is a one-to-one reciprocal continuous correspondence between the plane $V$ and the set of all real number pairs. In the present paper I establish the existence of such a correspondence. Under Theorem I, I show that every straight line in $V$ is a linear continuum. This proof is quite different from Lennes' proof of the above-mentioned theorem concerning continuous curves.

Theorem I. Every straight line in $V$ is a linear continuum.

I will prove this theorem by showing that every such line $l$ satisfies a set of five postulates for the linear continuum given by Veblen. $\dagger$ These postulates ( $A, B, C, D, E)$ are stated in terms of the relation $<$ (precedes). For the points of the line $l$ this relation may be defined in an obvious way in terms of the order (betweenness) of Veblen's axioms I-VIII, XI. It is then easy to show that, on the line $l, A, B$, and $D$ are satisfied. It remains to prove that $C$ and $E$ are satisfied. Postulates $C$ and $E$ are as follows.

C. Pseudo-Archimedean Postulate 1. If $\{P\}$ is an infinite set, then there exists a subset $\left[P_{\nu}\right]$, such that $P_{\nu}<P_{\nu+1}(\nu=1,2,3, \cdots)$, and if $P$ is any element of $\{P\}$ for which $P_{1}<P$, then there exists a such that $P<P_{\nu}$.

2. If $\{P\}$ is an infinite set, then there exists a subset $\left[P_{\nu}\right]$, such that $P_{\nu+1}^{\prime}<P_{v}^{\prime}$ $(\nu=1,2,3, \cdots)$, and if $P$ is any element of $\{P\}$ for which $P<P^{\prime}$, then there exists a $\nu$ such that $P_{\nu}^{\prime}<P$.

$E$. Postulate of Uniformity. For every element $P$ of a set $\{P\}$ which satisfies conditions $A, B, C, D$, and for every $\nu(\nu=1,2,3, \cdots)$ there exists a segment $\sigma_{\nu P}$ such that the set of segments $\left[\sigma_{\nu P}\right]$ has the following properties:

1. For a fixed $P, \sigma_{\nu P}$ contains $\sigma_{\nu+1 P}$.

2. For a fixed $P, P$ lies on every $\sigma_{\nu P}$ and is the only such element.

3. For every segment $\tau$, there exists $a \nu, \nu_{\tau}$, such that for no $P$ does $\sigma_{\nu_{\tau} P}$ contain $\tau$.

Proof of $C 1$. On $l$ there exist two points $A$ and $B$ such that $A<B$. There exists a point $O$ not on $l$ and a point $T$ such that $O T$ is parallel $\ddagger$ to $A B$. The ray from $T$ parallel to $O A$ must intersect the ray $A B$ in some point $P_{1}$. The ray from $T$ parallel to $O P_{1}$ intersects $A B$ in a point $P_{2}$. Continuing this process let $P_{\nu+1}$ denote the point in which $A B$ is cut by the ray from $T$ parallel

* N. J. Lennes, Curves in non-metrical analysis situs with an application in the calculus of variations, American Journal of M a thematics, vol. 33 (1911), pp. 285-326.

$\dagger \mathrm{O}$. Veblen, Definition in terms of order alone, etc., loc. cit.

$\ddagger$ In this paper the statement that $O T$ is parallel to $A B$ means that the ray $O T$ is parallel to the ray $A B$. This signifies that $O T$ itself fails to intersect $A B$ but $A B$ is intersected by every ray from $O$ that goes into the angle $T O A$. For a proof that $O T$ exists cf. Veblen, $A$ system of axioms for geometry, loc. cit., p. 370. It is to be observed that we are not assuming the parallel postulate. There may be infinitely many lines through $O$ which fail to cut the line $A B$. 
to $O P_{\nu}$. The set of points $\left[P_{\nu}\right]$ thus defined plays the part of $\left[P_{\nu}\right]$ in Veblen's $C 1$. This may be seen as follows: Suppose $P$ is any point such that $P_{1}<P$. If there should exist no $\nu$ such that $P<P_{\nu}$ then it may be easily seen with the belp of Postulate $\mathrm{B}^{*}$ that there would exist a point $P_{0}$ such that

(1) for every $\nu, P_{\nu}<P_{0}$,

(2) if $x<P_{0}$ then, for some $\nu, x<P_{\nu}$.

There would exist a ray through $O$ parallel to $T P_{0}$. This ray would cut $A B$ in a point $P^{\prime}$ such that $P^{\prime}<P_{0}$ and then there would exist $\nu_{1}$ such that $P^{\prime}<P_{\nu_{1}}$. It would clearly follow that $P_{0}<P_{\nu_{1+1}}$, which is contrary to part (1) of the above definition of $P_{0}$. Thus the supposition that there exists no $\nu$ such that $P<P_{\nu}$ leads to a contradiction. Postulate $\mathrm{C} 1$ is therefore established. Postulate $\mathrm{C} 2$ may be proved in an entirely analogous manner.

Proof of Postulate $E$. There exist two distinct points $A$ and $B$ on the line $l$ (Fig. 1). There exists a point $O$ not on $l$. There exist two rays $O C$ and $O D$

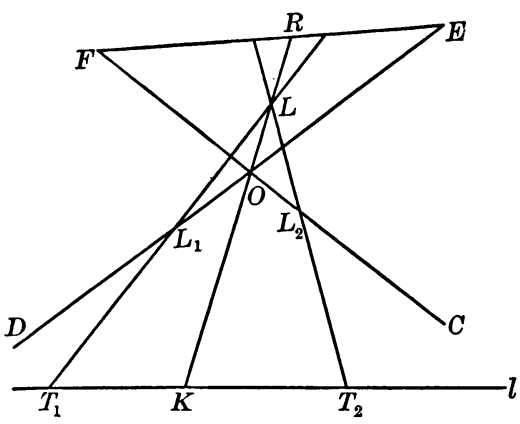

FIG. 1

parallel to $A B$ and $B A$ respectively. It may be easily proved with the aid of Postulate $B$ that there exist, on the rays $O C$ and $O D$ respectively, two sets of points, $\left[P_{\nu}\right]$ and $\left[P_{\nu}^{\prime}\right]$, such that, for every $\nu, P_{\nu} P_{v+1} O, \dagger P_{\nu}^{\prime} P_{\nu+1}^{\prime} O$, and such that $\operatorname{Lim} \ddagger P_{\nu}=O$ and $\operatorname{Lim} P_{\nu}^{\prime}=O$. There exist points $E$ and $F$ such that $D O E$ and $C O F$. Let us suppose that $O C$ and $O D$ are not collinear. $\S$ Then if $P$ is any point on $l$ the line $P O$ meets the segment $F E$ in a point $S$. It is clear that $S P_{\nu}$ and $S P_{\nu}^{\prime}$ must cut $l$. Let $S_{\nu}$ and $S_{\nu}^{\prime}$ respectively denote the points in which they cut it. Let $\sigma_{\nu P}$ denote the segment $S_{\nu} S_{\nu}^{\prime}$. This segment plays the rôle of $\sigma_{\nu P}$ in Postulate $E$. That this is true as far as $E 1$

* As stated above, Postulate $B$ is a theorem here.

$\dagger A B C$, used as a sentence, signifies that $B$ is between $A$ and $C$.

$\ddagger \operatorname{Lim} P_{\nu}=O$ signifies that if $k$ is any segment of the line $O D$ containing $O$ in its interior then there exists a positive number $\delta$ such that if $\nu>\delta$ then $P_{\nu}$ lies within $k$.

\& If $O C$ and $O D$ are collinear, replace the segment $F E$ by the broken line $F Z E$ where $Z$ is a point on the remote side of $O C$ from $l$. 
and $E 2$ are concerned is evident. That $E 3$ also is satisfied may be proved as follows. Let $\tau$ be any segment on $l$. Let $T_{1}$ and $T_{2}$ (where $T_{1}<T_{2}$ ) denote its end points. There exists a point $K$ such that $T_{1} K T_{2}$. The line $K O$ intersects the segment $F E$ in a point $R$. There exists a point $L$ between $O$ and $R$. The segment $T_{1} L$ intersects the ray $O D$ in a point $L_{1}$ and $T_{2} L$ intersects $O C$ in a point $L_{2}$. Let $\nu_{\tau}$ be the smallest $\nu$ for which $P_{\nu}$ lies between $L_{1}$ and $O$ and $P_{\nu}^{\prime}$ lies between $L_{2}$ and $O$. It is not difficult to see that this $\nu_{\tau}$ plays the part of $\nu_{\tau}$ in Postulate E3. Thus E3 is proved and Theorem I is established.

Definition.* In the plane $V$ the point $P$ is said to be a limit point of the point set $S$ if every triangle containing $P$ in its interior contains at least one point of $S$ different from $P$.

Theorem II. Between the plane $V$ and an ordinary euclidean plane $E$ there is a one-to-one reciprocal continuous $\dagger$ correspondence of points.

Proof. Let $l$ be a line lying in the plane $V$. By Theorem I the set of points that constitute this line may be brought into a one-to-one continuous correspondence with the set of all real numbers. Let a definite correspondence of this type be set up. The number which corresponds to a point on $l$ will be called the abscissa of that point. Let $O$ (Fig. 2) be the point whose abscissa

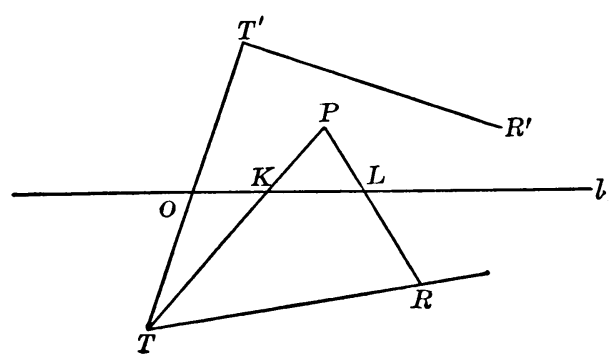

Fig. 2

is zero, and $I$ the point whose abscissa is 1 . There exists a point $T$ not on $l$. There exists a point $T^{\prime}$ such that $O$ is between $T$ and $T^{\prime}$. There exist rays $T R$ and $T^{\prime} R^{\prime}$ parallel to $O I$. If $P$ is any point on the opposite side of $O I$ from $T$ then $P T$ and $P R$ will intersect the line $O I$. Let $K$ and $L$ respectively denote the points in which they intersect it. The abscissa of $P$ is defined as the abscissa of $K$ and the ordinate of $P$ is defined as $\overline{K L}$. $\ddagger$ If $P$ is on the opposite side of $O I$ from $T^{\prime \prime}$ then $P T^{\prime}$ and $P R^{\prime}$ will intersect $O I$ in points $K^{\prime}$

* Cf. O. Veblen, Theory of plane curves in non-metrical analysis situs, loc. cit., p. 85.

$\dagger$ The statement that such a correspondence is continuous signifies that if a point $P$ in $V$ is a limit point of a point set $S$ lying in $V$, then $P^{\prime}$ is a limit point of $S^{\prime}$, and conversely, where $P^{\prime}$ and $S^{\prime}$ are the point and point set in $E$ which correspond to $P$ and $S$ respectively.

$\ddagger$ The symbol $\overline{K L}$ is used to denote the number obtained by subtracting the abscissa of $K$ from that of $L$. 
and $L^{\prime}$ respectively. In this case the abscissa of $P$ is defined as the abscissa of $K^{\prime}$ but the ordinate of $P$ is defined as $\overline{L^{\prime} K^{\prime}}$. In accordance with these definitions every point $P$ in $V$ has an abscissa $x$ and an ordinate $y .^{*}$ It is not true however that to every real number pair $(x, y)$ there corresponds a point in $V$ whose coördinates are $x$ and $y$. Indeed if $P$ is a point on the remote side of $O I$ from $T$ then it is clear that the ray through $R$ which is parallel to $T P$ must cut $O I$ in some point $N$ and that $\overline{K N}$ is the least upper bound of the ordinates of all points whose common abscissa equals the abscissa of $P$. Now $\overline{K N}$ is a function of $x$, the abscissa of $P$. Let us write

$$
\overline{K N}=f(x) \text {. }
$$

Similarly let $F(x)$ denote the greatest lower bound of the ordinates of all points with a given abscissa $x$ which lie on the same side of $O I$ as $T$. Now, using a rectangular system of coördinates in an ordinary euclidean plane, plot the two curves $y=f(x)$ and $y=F(x)$. The first of these curves will lie entirely above, and the second will lie entirely below, the $x$-axis. Let $H$ denote the region lying between them. To each point $P$ in $V$ there corresponds the point of $H$ whose rectangular coördinates are the $V$-coördinates of $P$. It is easy to see that the correspondence thus established between $V$ and $H$ is continuous. But $H$ is clearly a simply connected region. Hence it may be brought into a one-to-one continuous correspondence with the whole plane $E$. It follows that there exists such a correspondence between $V$ and $E$. Thus Theorem II is established.

In a definite correspondence of this type between $V$ and $E$, each straight line in $E$ corresponds to some continuous curve in $V$. It is clear that if the set $S^{\prime}$ of all curves in $V$ that correspond to straight lines in $E$ are themselves looked upon as straight lines, then

Theorem III. With respect to this new choice of straight lines $V$ is an ordinary euclidean plane.

The following is an equivalent statement in different form.

Theorem IV. In the plane $V$ there exists a set $S$ of simple continuous curves (each of which contains all its limit points) such that

(1) Each S-curve $\dagger$ divides the plane $V$ into two parts. +

(2) Through any two given points of $V$ there is one and only one $S$-curve.

(3) If $s$ is an $S$-curve and $P$ is a point in $V$ not lying on $s$ then through $P$ there is only one S-curve that fails to have a point in common with $s$.

* Let us call these coördinates the $V$-coördinates of the point $P$.

$\dagger$ By an $S$-curve is meant a curve of the set $S$.

¥The statement that the curve $s$ divides the plane $V$ into two parts signifies that the point-set $V-s$ consists of two mutually exclusive subsets $V_{1}$ and $V_{2}$ such that every two points in $V_{i}(i=1,2)$ can be joined by a jordan arc which contains no point of $s$, but no point of $V_{1}$ can be so joined to a point of $V_{2}$. 
(4) The set of curves $S$ satisfies Desargues Theorem.*

It may be seen as follows that every set $S$ satisfying the conditions stated in Theorem IV must also be a set $S^{\prime} \dagger$ satisfying Theorem III. Suppose that $s_{1}$ and $s_{2}$ are two $S$-curves having a point $O$ in common. Suppose that $s_{1}$ does not cross $s_{2}$ at $O$. Then it is clear $\ddagger$ that $O$ divides $s_{1}$ into two half-curves $O A$ and $O B$ and $s_{2}$ into two half-curves $O C$ and $O D$ such that $O D$ and $O A$ are separated from each other by $O B$ and $O C$. The $S$-curve which joins $A$ to $D$ must then either cross the half-curve $O B$ at a point $E$ or cross the halfcurve $O C$ at a point $F$. The point $E$ would, of necessity, be $\operatorname{distinct}$ from $A$ and $F$ would be distinct from $D$. Thus in one case the $S$-curve $A D$ would have two distinct points $A$ and $E$ in common witb $s_{1}$ and in the other case it would have two distinct points $D$ and $F$ in common with $s_{2}$. Hence both cases are contrary to condition (2) of Theorem IV. Therefore two S-curves with a point in common must cross each other at that point. It easily follows that Veblen's Triangle Transversal Axiom VIII holds true for the set of curves $S$. That his Axioms I-VII, XI, and XII hold true for $S$ is clear. With the help of condition (4) of Theorem IV it follows $\|$ that the plane $V$ may be regarded as lying in a 3-dimensional space $R$ in which all of Veblen's Axioms I-XII are valid, the "straight lines" of $R$ that lie in $V$ being the $S$-curves. Hence the plane $V$ is euclidean with respect to these curves as straight lines.

The original "straight lines" of $V$ satisfy conditions (1) and (2) of Theorem IV, but they do not necessarily satisfy condition (3) or condition (4).

The University of Pennsyluania.

* Cf. D. Hilbert, Foundations of Geometry, Townsend's translation, §22.

†Cf. above.

$\ddagger$ That no $S$-curve is closed can easily be proved with the aid of conditions (1) and (2).

§ O. Veblen, $A$ system of axioms for geometry, loc. cit.

\|Cf. Hilbert, loc. cit.

T Cf. Hilbert, loc. cit., and F. R. Moulton, A simple non-desarguesian plane geometry, these Transactions, vol. 3 (1902), p. 192. 